\title{
Neuropathology of supercentenarians - four autopsy case studies
}

\author{
Masaki Takao ${ }^{1,4^{*}}$, Nobuyoshi Hirose ${ }^{2}$, Yasumichi Arai ${ }^{2}$, Ban Mihara ${ }^{3}$ and Masaru Mimura ${ }^{4}$
}

\begin{abstract}
Supercentenarians (aged 110 years old or more) are extremely rare in the world population (the number of living supercentenarians is estimated as 47 in the world), and details about their neuropathological information are limited. Based on previous studies, centenarians (aged 100-109 years old) exhibit several types of neuropathological changes, such as Alzheimer's disease and Lewy body disease pathology, primary age-related tauopathy, TDP-43 pathology, and hippocampal sclerosis. In the present study, we provide results from neuropathological analyses of four supercentenarian autopsy cases using conventional and immunohistochemical analysis for neurodegenerative disorders. In particular, we focused on the pathology of Alzheimer's disease and Lewy body disease, as well as the status of hippocampal sclerosis, TDP-43 pathology, aging-related tau astrogliopathy, and cerebrovascular diseases. Three cases were characterized as an "intermediate" level of Alzheimer's disease changes (NIA-AA guideline) and one was characterized as primary age-related tauopathy. TDP-43 deposits were present in the hippocampus in two cases. Neither Lewy body pathology nor hippocampal sclerosis was observed. Aging-related tau astrogliopathy was consistently observed, particularly in the basal forebrain. Small vessel diseases were also present, but they were relatively mild for cerebral amyloid-beta angiopathy and arteriolosclerosis. Although our study involved a small number of cases, the results provide a better understanding about human longevity. Neuropathological alterations associated with aging were mild to moderate in the supercentenarian brain, suggesting that these individuals might have some neuroprotective factors against aging. Future prospective studies and extensive molecular analyses are needed to determine the mechanisms of human longevity.
\end{abstract}

Keywords: Aging, Supercentenarian, Neuropathology, Amyloid-beta, Tau, TDP-43

\section{Introduction}

Increased human longevity is a goal in many parts of the world. Although it is difficult to clearly define "successful aging," aging without any severe diseases or disabilities is ideal. Among the many factors associated with a decreased ability to function during human aging, brain disease is an important factor. According to epidemiological data, the number of centenarians (aged 100 or more) in the world was 451,000 in 2015 (http://esa.un.org/unpd/wpp/Download/Standard/Population; World Population Prospects, 2015 revision). Additionally, this number could increase to 3,678,000 by 2050 (http://esa.un.org/unpd/wpp/Download/ Standard/Population; World Population Prospects, 2015

\footnotetext{
* Correspondence: msktakaobrb@gmail.com

'Department of Neurology, Saitama Medical University International Medical Center, Yamane, 1397-1 Yamane, Hidaka, Saitama 350-1298, Japan

${ }^{4}$ Department of Neuropsychiatry, School of Medicine, Keio University, 35

Shinanomachi, Shinjuku-ku, Tokyo 160-8582, Japan

Full list of author information is available at the end of the article
}

revision). Neuropathological analyses of centenarian brains remain limited. From a Japanese cohort, centenarian brains exhibited Alzheimer's disease (AD) and cerebrovascular disease (CVD), as well as cases with no definite pathological alterations [1]. Based on our introductory analysis of 58 centenarian brains (mean age $101.5 \pm 1.7 \mathrm{y}$ ), a high likelihood of intermediate AD pathology (NIA Reagan criteria) was observed in 15 cases $(25.8 \%)$ and a low likelihood of sparse neuritic plaques and stage I/II of Braak staging of neurofibrillary tangles was observed in 8 cases (13.7 \%). Additionally, 21 cases (36.2 \%) were characterized as primary age-related tauopathy (PART), which was recently recognized as a neuropathological condition of the aging brain. Recent published neuropathological data from three cohorts in the US and UK showed that AD pathology is not common [2]. Hippocampal sclerosis-related aging and Lewy body pathology were reported, and arteriolosclerosis was shown to be possibly associated with hippocampus 
sclerosis-related aging [2, 3]. Therefore, those centenarian cases may exhibit high proportions of $\mathrm{AD}$ pathology, as well as non-AD pathology. However, the average age of individuals analyzed in those studies was close to 100 years. In autosomal-dominant cases of $\mathrm{AD}$, the onset of amyloidbeta deposition might be 15 years prior to the onset of clinical symptoms [4]. Therefore, centenarians close to 100 years of age with $\mathrm{AD}$ pathology might represent individuals who developed pathological $\mathrm{AD}$ changes between 80 and 90 years of age.

Compared with centenarians, supercentenarians (aged 110 years old or more) are extremely rare in the human race. According to a study by the "Gerontology Research Group" in 2016, the number of living supercentenarians is estimated as 47 in the world (http://supercentenarianresearch-foundation.org/TableE.aspx, accessed July 14, 2016). However, it is difficult to estimate the actual number of worldwide supercentenarians, because some individuals lack a birth certificate or, depending on the country of origin, it might be difficult to obtain a reliable birth certificate. Therefore, systematic neuropathological analysis of supercentenarian brains remains difficult. We have attempted to locate supercentenarians and communicate with their families to obtain brain samples for pathological analyses. Clarification of the neuropathological conditions of these exceptional humans might provide a better understanding of the pathomechanisms involved in human longevity.

A previous clinical and neuropathological study of a 115-year-old woman from the Netherlands [5] reported well-preserved cognitive function, with only mild aging alterations. In the present study, we provide results from neuropathological analyses of four supercentenarian autopsy cases using conventional and immunohistochemical analysis for neurodegenerative disorders. In particular, we focused on the pathology of AD and Lewy body disease, as well as the status of hippocampal sclerosis, TDP-43 pathology, aging-related tau astrogliopathy (ARTAG), and CVD.

\section{Materials and methods}

\section{Clinical information}

All individuals were contacted by one $(\mathrm{NH})$ of the authors prior to death. At that time, clinical conditions and some medical anamnesis were obtained from each individual and their relatives, as well as their caregivers. Blood samples were obtained for future analyses. To confirm the age of individuals, the date of birth was determined using the family register. Apolipoprotein E (APOE) genotyping was also determined.

\section{Autopsy}

Because the individuals were placed in elderly care facilities, the bodies were transferred from the facilities to our brain bank by hospital ambulance following death. At the time of autopsy, fresh brain tissue was dissected at the mid-sagittal line. The right cerebrum, cerebellum, and brainstem were immediately frozen by dry ice and stored at $-80{ }^{\circ} \mathrm{C}$ for future studies. The left hemisphere of the brain was fixed in $20 \%$ neutral-buffered formalin (Wako, Osaka, Japan) for neuropathological analysis.

\section{Neuropathological studies}

Neuropathological studies were performed on postmortem tissue from four individuals (Table 1). All cases were registered with our Brain Bank and neuropathologically analyzed according to the following protocols. Samples were dissected from coronal slices of fixed brains from the following regions: superior and middle frontal gyri, anterior cingulate gyrus, superior and middle temporal gyri, motor and sensory cortices, insular cortex, calcarine cortex, amygdala, hippocampus, subiculum, parahippocampal gyrus, caudate nucleus, putamen, globus pallidus, thalamus, cerebellum, midbrain, pons, and medulla [6,7]. Blocks were dehydrated in alcohol gradients, cleared in xylene, and embedded in paraffin. The brain tissues were cut into $6-\mu \mathrm{m}-$ thick sections. The sections were stained with hematoxylin and eosin (HE), Klüver-Barrera for myelin, and modified Gallyas-Braak silver staining for fibrils. For immunohistochemical studies, monoclonal antibodies specific to amyloid A $\beta$ (11-28) (12B8, 1:100, IBL, Gunma, Japan), phospho-tau (AT8, 1:3000, Innogenetics, Ghent, Belgium), 4-repeat isoform tau (RD4, 1:500, 1E1/A6, Merck Millipore, Darmstadt, Germany), 3-repeat isoform tau (RD3, 1:500, 8E6/C11, Merck Millipore), phosphorylated $\alpha$-synuclein (1:7000, pSyn\#64, monoclonal, Wako), and phosph-TDP-43 (s409/ 410, 1:7000, COSMO BIO, Tokyo, Japan) were used. The sections were processed using a Ventana Discovery automated immunostainer (Roche, Basel, Switzerland), and the sections were counterstained with hematoxylin.

For neuropathological diagnosis of Alzheimer's disease, we used the Consortium to Establish a Registry for Alzheimer's Disease (CERAD) methodologies, widely applied to make a diagnosis of Alzheimer's disease, as a guide for analyzing the frequency of neuritic plaques [8]. Braak staging of neurofibrillary tangle formation and Thal phase of amyloid beta were also used [9-11]. Eventually, National Institute on Aging (NIA)-Reagan criteria (1997) and NIA-Alzheimer's Association (NIA-AA) guidelines (2012) were applied for determining the level of AD pathological change [12, 13]. Those neuropathological strategies also allowed us to identify whether each case corresponded with PART criteria.

The ARTAG type and anatomical areas were analyzed in all sections according to a recently published harmonized evaluation strategy using AT8 immunohistochemistry [14]. First, AT8 immunoreactive thorn-shaped astrocytes (TSA) and fine granular immunoreactivity 
Table 1 Patient demographics and summary of neuropathological analyses

\begin{tabular}{|c|c|c|c|c|}
\hline & Case 1 (111 yo) & Case 2 (111 yo) & Case 3 (114 yo) & Case 4 (110 yo) \\
\hline Sex & Female & Female & Female & Female \\
\hline Past medical history & HT-, DM- & HT-, DM- & HT-, DM- & $\mathrm{HT}+, \mathrm{DM}-$ \\
\hline $\begin{array}{l}\text { Clinical condition before } \\
\text { death }\end{array}$ & Clear communication, wheel chair & No dementia & $\begin{array}{l}\text { Clear communication, } \\
\text { wheel chair }\end{array}$ & $\begin{array}{l}\text { Almost clear communication, } \\
\text { wheel chair }\end{array}$ \\
\hline $\begin{array}{l}\text { Barthel index } \\
\text { CDR } \\
\text { MMSE }\end{array}$ & $\begin{array}{l}10 \\
0.5 \\
\text { NA due to refusal } \\
\text { Age } 106\end{array}$ & $\begin{array}{l}60 \\
\text { NA } \\
15 \\
\text { Age } 106\end{array}$ & $\begin{array}{l}90 \\
0 \\
22 \\
\text { Age } 108 \\
\text { MMSE was } 18 \text { at age } \\
113 \text { and } 114\end{array}$ & $\begin{array}{l}20 \\
\text { NA } \\
\text { NA } \\
\text { Age } 109\end{array}$ \\
\hline Cause of death & Heart failure & Renal failure & Senility & Sepsis \\
\hline APOE & $2 / 3$ & $2 / 3$ & $3 / 3$ & $3 / 3$ \\
\hline Brain weight (fresh) & 460 (left hemisphere) & 925 & 1,015 & 1,115 \\
\hline Atrophy & $F, T$ & $F, T$ & $F, T$ & $\mathrm{~T}$ \\
\hline A-beta, Thal phase & $3(A 2)$ & $3(A 2)$ & $1(\mathrm{~A} 1)$ & $2(\mathrm{~A} 1)$ \\
\hline NFT stage (AT8) (Braak) & III (B2) & IV (B2) & III (B2) & IV (B2) \\
\hline Neuritic plaques (CERAD) & Moderate (C2) & Moderate (C2) & Sparse (C1) & Moderate (C2) \\
\hline CAA & None & None & None & Mild \\
\hline $\begin{array}{l}\text { AD pathological changes } \\
\text { (NIA-Reagan) }\end{array}$ & Intermediate & Intermediate & Unclassified & Intermediate \\
\hline $\begin{array}{l}\text { AD pathological changes } \\
\text { (NIA-AA) }\end{array}$ & Intermediate & Intermediate & $\begin{array}{l}\text { Low } \\
\text { PART possible }\end{array}$ & Intermediate \\
\hline ARTAG & $\begin{array}{l}\text { Subpial, subependymal, gray matter, } \\
\text { white matter, perivascular }\end{array}$ & $\begin{array}{l}\text { Subpial, gray matter, } \\
\text { white matter }\end{array}$ & $\begin{array}{l}\text { Subpial, gray matter, } \\
\text { white matter }\end{array}$ & $\begin{array}{l}\text { Subpial, subependymal, gray } \\
\text { matter, perivascular }\end{array}$ \\
\hline Arteriolosclerosis & Mild to moderate & Mild to moderate & Mild to moderate & Mild to moderate \\
\hline White matter rarefaction & Moderate & Mild & Mild & Moderate \\
\hline $\begin{array}{l}\text { État criblé (basal ganglia } \\
\text { and thalamus) }\end{array}$ & Moderate & Severe & Severe & Moderate \\
\hline Vascular brain injury & Multiple cortical infarcts & None & None & None \\
\hline $\begin{array}{l}\text { Alpha-synuclein } \\
\text { pathology }\end{array}$ & None & None & None & None \\
\hline TDP-43 pathology & Present, subiculum, PHG & $\begin{array}{l}\text { Present, subiculum, } \\
\text { PHG }\end{array}$ & Uncus, sparse & Uncus, sparse \\
\hline Hippocampal sclerosis & None & None & None & None \\
\hline $\begin{array}{l}\text { Hirano bodies/GVD } \\
\text { (Hippocampus) }\end{array}$ & Present/present & Present/present & Present/present & Present/present \\
\hline
\end{tabular}

Abbreviations: ARTAG aging-related tau astrogliopathy, CAA cerebral amyloid angiopathy, CDR Clinical Dementia Rating, CERAD Consortium to Establish a Registry for Alzheimer's Disease, DM diabetes mellitus, $F$ frontal lobe, GVD granulovaculaor degeneration, $H T$ hypertension, MMSE Mini-Mental State Examination, NA not available, NIA National Institute on Aging, NIA-AA NIA-Alzheimer's Association, $T$ temporal lobe, yo years old

(GFA) were identified. Second, the type, major anatomical involvement, severity, and detailed regional distribution were determined [14]. TDP-43 immunoreactivity was classified as neuronal cytoplasmic (NCI), neuronal nuclear (NNI), and glial cytoplasmic (GCI) immunoreactive deposits, as well as neurites.

Cerebral amyloid angiopathy and arteriolosclerosis, as well as white matter rarefaction and état criblé, were assessed using a subjective scale of none, mild, moderate or severe.

We obtained written informed consent from the relatives of the deceased for autopsy and further neuropathological analysis, and all subjects were registered with our brain bank for future research. The brain bank was approved by the Ethics Committee of Mihara Memorial Hospital for neuropathological analysis.

\section{Results}

\section{Clinical information}

All four supercentenarian individuals were female. Because they were placed in elderly care facilities, the individuals did not receive extensive medical treatment during the end stages of life. It was difficult to determine whether they were demented at the time of interview. 
Case 1 was able to communicate and move her wheelchair herself. In Case 2, no apparent dementia symptoms were reported by the nursing staff. Cases 3 and 4 were able to communicate with other people and manipulate their wheel chairs. In some individuals, Barthel index, clinical dementia rating (CDR) scale and Mini-Mental State Examination (MMSE) were evaluated (Table 1). The clinical characteristics are summarized in Table 1.

\section{Neuropathology}

Because brain pathology of supercentenarians has not been previously well described, we report descriptive neuropathological findings from each case. The summary of neuropathological findings is shown in Table 1.

\section{Case 1}

\section{Gross neuropathology}

The fresh brain weight of the left hemisphere was 460 grams. Unfortunately, the right cerebral hemisphere (frozen side) was not weighed at the time of autopsy. After formalin fixation, there was mild atrophy in the frontal and temporal lobes (Figs. 1a and 2a). Atherosclerosis was mild in the major cerebral arteries. No atheromatous plaques were seen in the leptomeningeal vessels. On the coronal sections, a mild enlargement was present at the posterior part of lateral ventricle. No atrophy was seen in the cerebellum. The substantia nigra and locus coeruleus were well pigmented.

\section{Microscopic neuropathology}

Neuronal loss and gliosis was none to mild in the majority of the cerebral cortex. Neurons in the substantia nigra and locus coeruleus were well preserved. Moderate numbers of $\mathrm{A}$-immunoreactive neuritic plaques were observed using CERAD methodology. Aß-immunoreactive parenchymal deposits were characterized as phase 3 using Thal's methodology. There was also a moderate level of Aß-immunoreactive diffuse plaques observed, although no $A ß-$ immunoreactive cerebral amyloid angiopathy was seen. AT8-immunoreactive NFTs were stage III according to Braak methodology (Figs. 3a and 4a, Table 1). Therefore, Case 1 was assigned an intermediate level of $\mathrm{AD}$ pathological changes according to NIA-Reagan and NIA-AA criteria. Alpha-synuclein immunoreactive deposits, as well as AT8-immunoreactive tufted astrocytes and astrocytic plaques, were not observed. All ARTAG types were mainly observed in the medal temporal lobe and subcortical areas. The perivascular ARTAG pattern was particularly present in the basal forebrain (Figs. 5 and 7). No hippocampal sclerosis was observed. TDP-43-immunoreactive NCIs and neuritis were present in the basal forebrain, hippocampus, and subiculum (Fig. 6, Table 2). NNIs and GCIs were also present (Table 2). In the parietal cortex, there was a small area with neuronal loss, with gliosis and hemosiderin-laden macrophages in the cortex. These findings were consistent with an old hemorrhagic infarct. A small vessel occluded by an organizing thrombus was seen in the subarachnoid space immediately adjacent to the infarct. Because there were no atherosclerotic changes in the leptomeningeal vessels, the infarct was considered to be an embolic lesion. Mild to moderate arteriolosclerosis was observed in various areas (Table 3). Mineralization was seen in the small vessel wall in the globus pallidus (Fig. 9a).

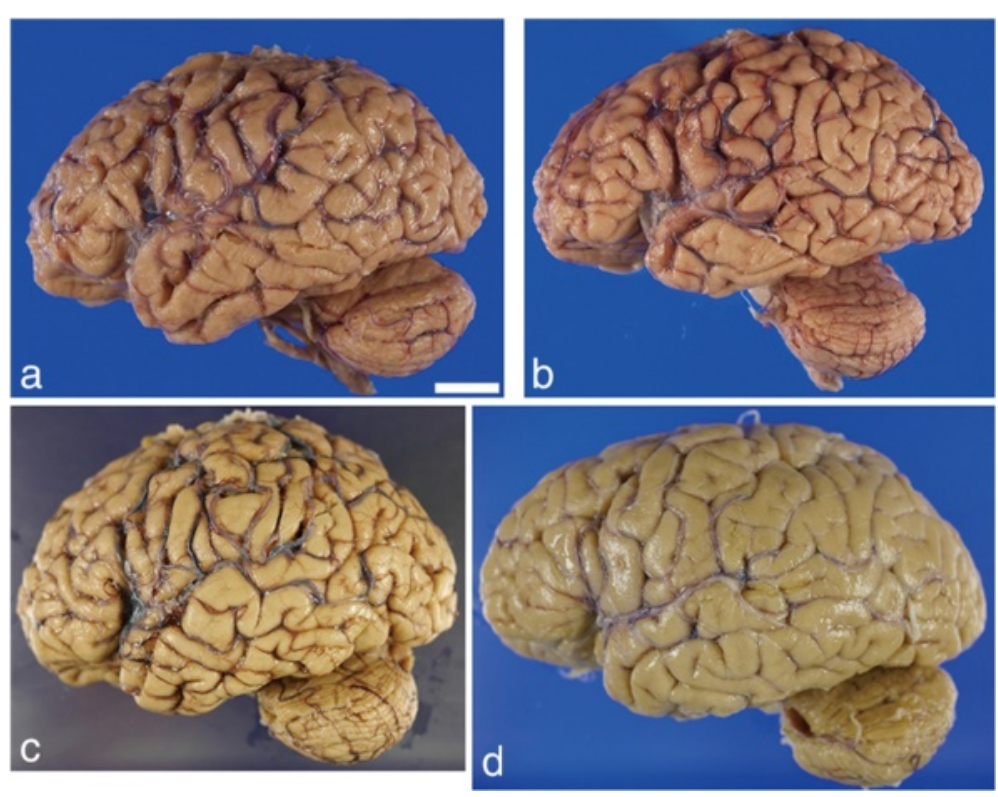

Fig. 1 Photomicrographs of fixed left-brain hemispheres from four supercentenarians. Mild atrophy is present in the frontal and/or temporal lobes. a Case 1, (b) Case 2, (c) Case 3, (d) Case 4. Bar $=2 \mathrm{~cm}$ 

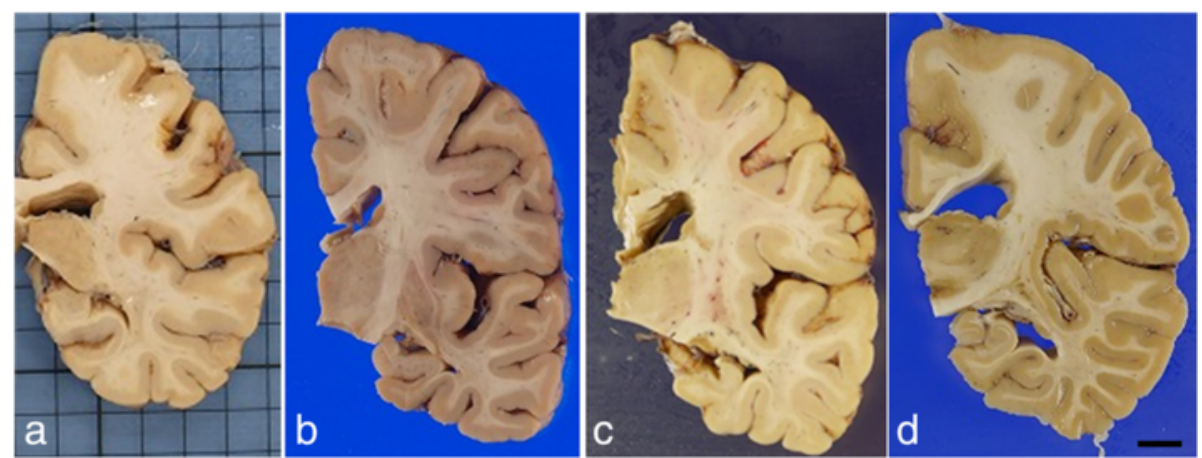

Fig. 2 Coronal sections at the basal ganglia and hippocampus level. Cortical ribbons and cerebral white matter are well-preserved. Small cortical infarct in Case 1 (a). a Case 1, (b) Case 2, (c) Case 3, (d) Case 4. Bar $=1 \mathrm{~cm}$

\section{Case 2}

\section{Gross neuropathology}

The fresh brain from Case 2 weighed 925 grams. After formalin fixation, there was mild atrophy in the frontal lobe and moderate atrophy in the temporal lobes (Fig. 1b). Atherosclerosis was mild in the major cerebral arteries. No atheromatous plaques were seen in the leptomeningeal vessels. On coronal sections, there were no focal lesions except mild enlargement of the lateral ventricle (Fig. 2b). Subcortical nuclei were well preserved without obvious atrophy. No atrophy was seen in the cerebellum. The substantia nigra and locus coeruleus were well pigmented.

\section{Microscopic neuropathology}

Neuronal loss and gliosis was none to mild in the majority of the cerebral cortex. Neurons in the substantia nigra and locus coeruleus were well preserved. Levels of Aß-immunoreactive neuritic plaques and parenchymal deposits were determined moderate by CERAD and phase 3 by Thal's staging methodology, respectively. There were also sparse levels of $A ß$-immunoreactive diffuse plaques observed, but no Aß-immunoreactive cerebral amyloid angiopathy was observed. AT8-immunoreactive NFTs were stage IV according to Braak methodology. Therefore, Case 2 was assigned to an intermediate level of $\mathrm{AD}$ pathological changes according to NIA-Reagan and NIA-AA criteria

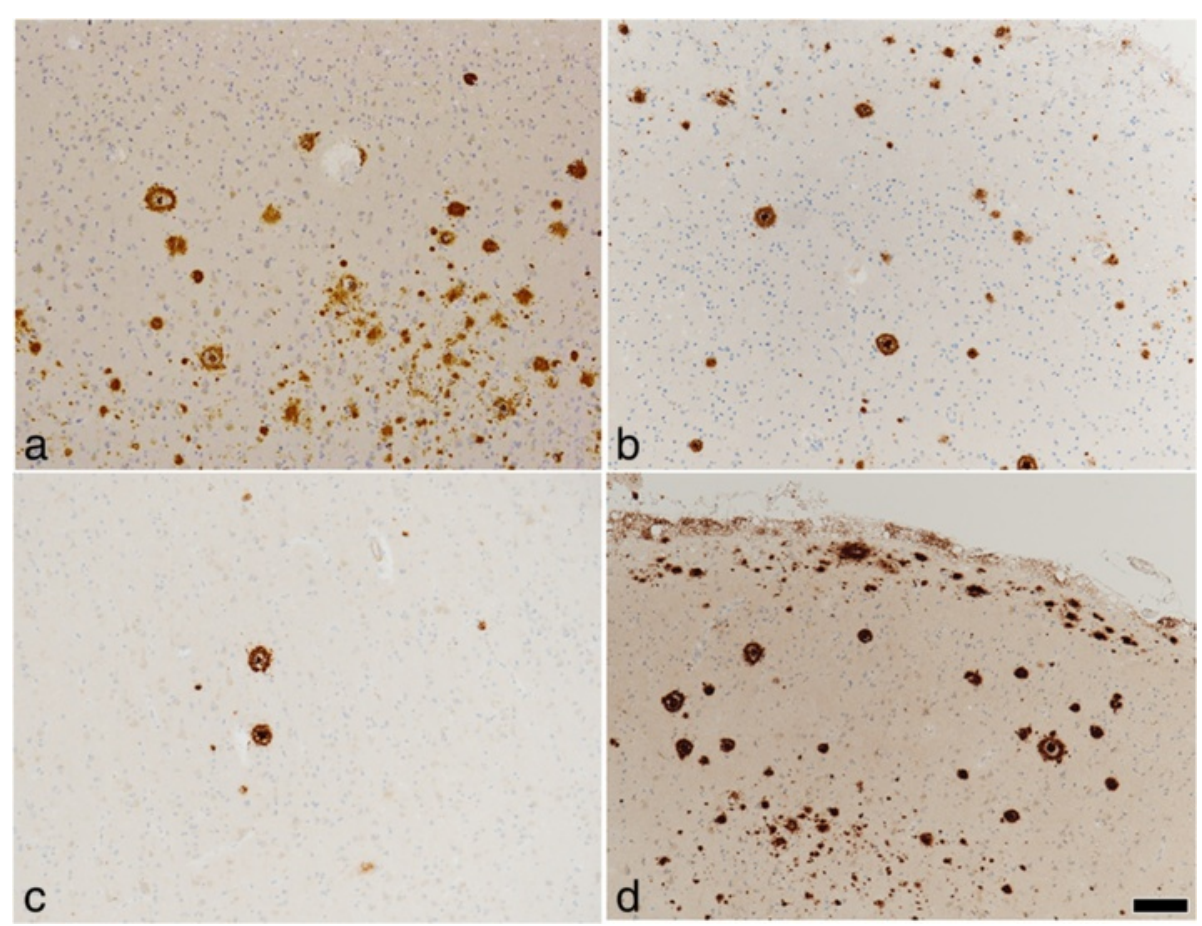

Fig. 3 Photomicrographs of the temporal cortices from four cases. Immunohistochemistry using monoclonal antibody specific to Aß (11-28). a Case 1, (b) Case 2, (c) Case 3, (d) Case 4. Bar $=100 \mu \mathrm{m}$ 


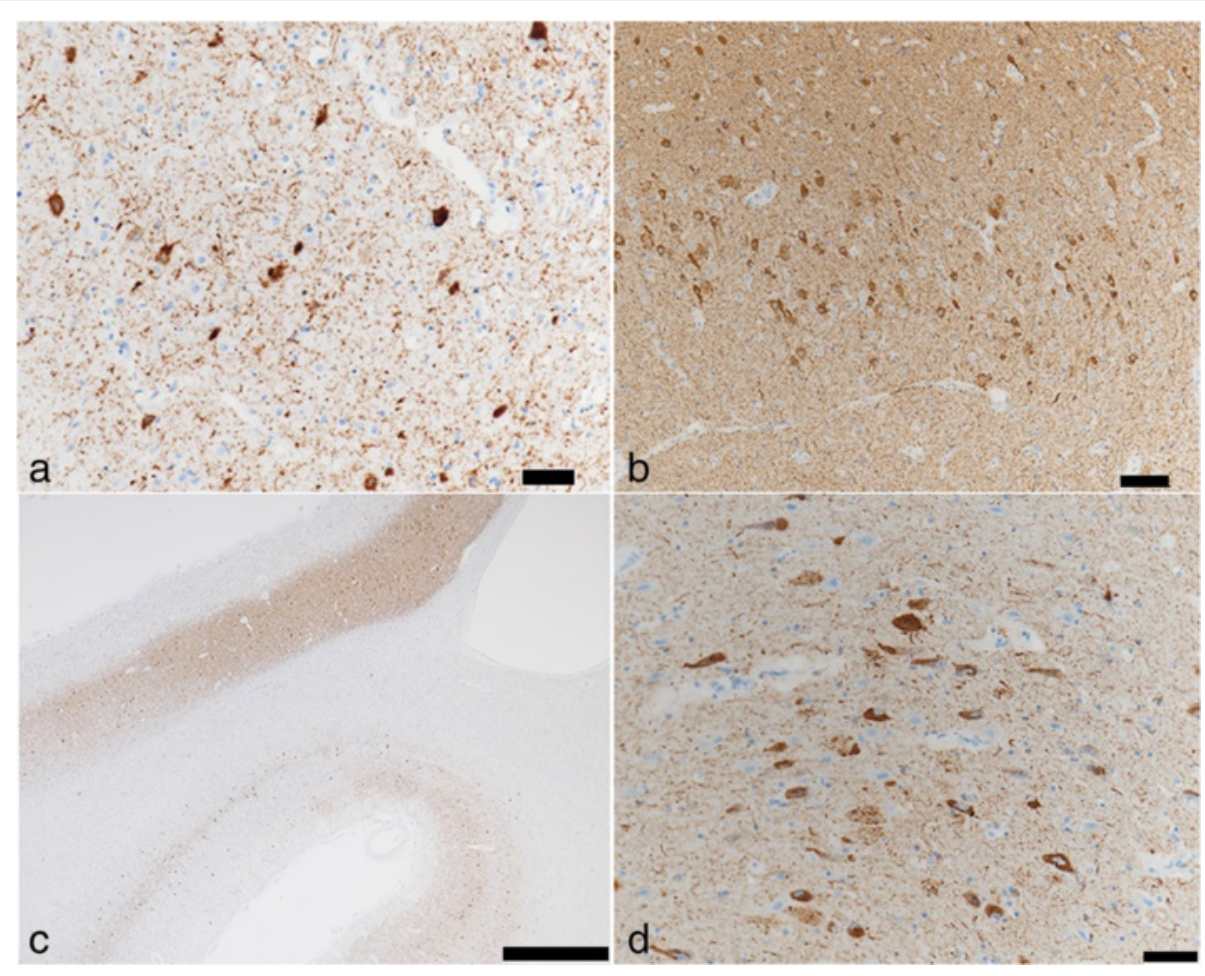

Fig. 4 Photomicrographs of hippocampus and parahippocampus from four cases. Immunohistochemistry using monoclonal antibody specific to p-tau (AT8). a Case 1, (b) Case 2, (c) Case 3, (d) Case 4. Bar $=50 \mu \mathrm{m}$

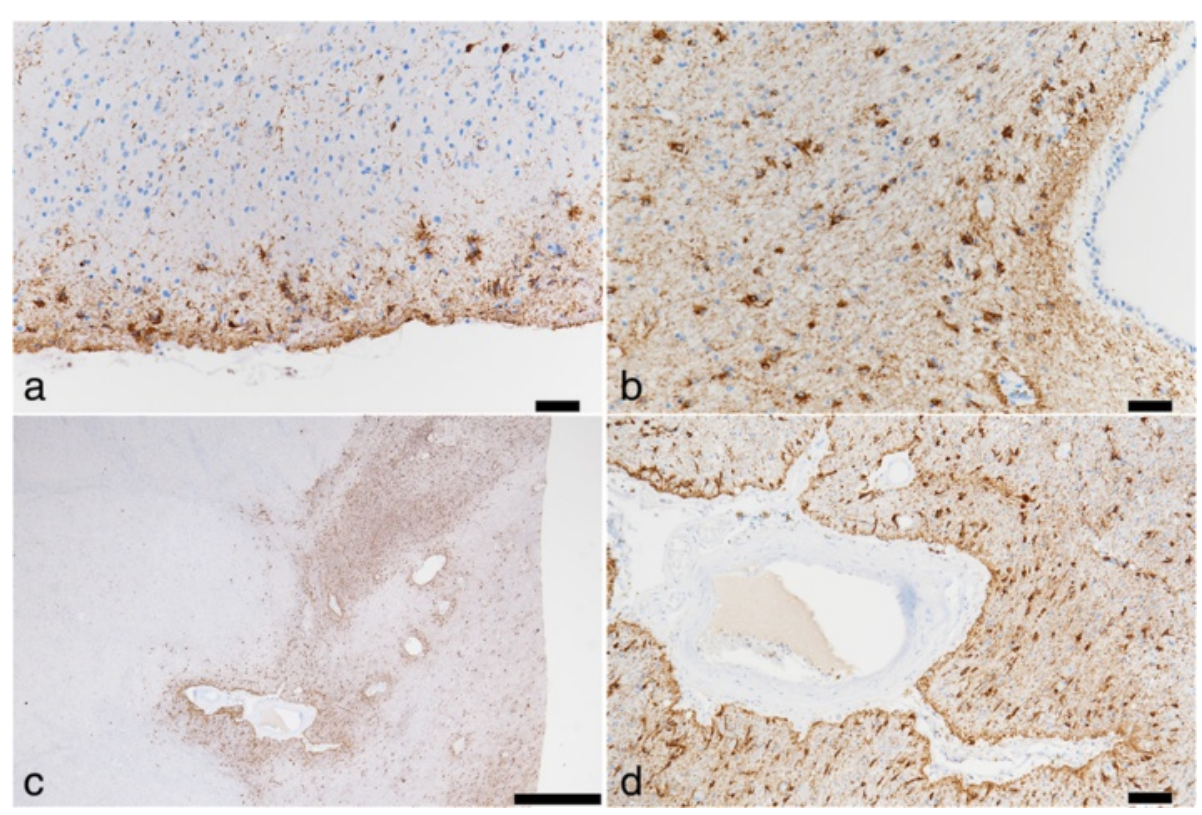

Fig. 5 ARTAG of Case 1. ARTAG (thorn-shaped astrocytes) is present in the subpial areas of inferior temporal gyrus (a), white matter close to the lateral ventricle of the hippocampus (b), and perivascular area of the basal forebrain (c, d). Immunohistochemistry using monoclonal antibody specific to $\mathrm{p}$-tau (AT8). Bar $=50 \mu \mathrm{m}(\mathbf{a}, \mathbf{b}, \mathbf{d})$. Bar $=1000 \mu \mathrm{m}$ (c) 


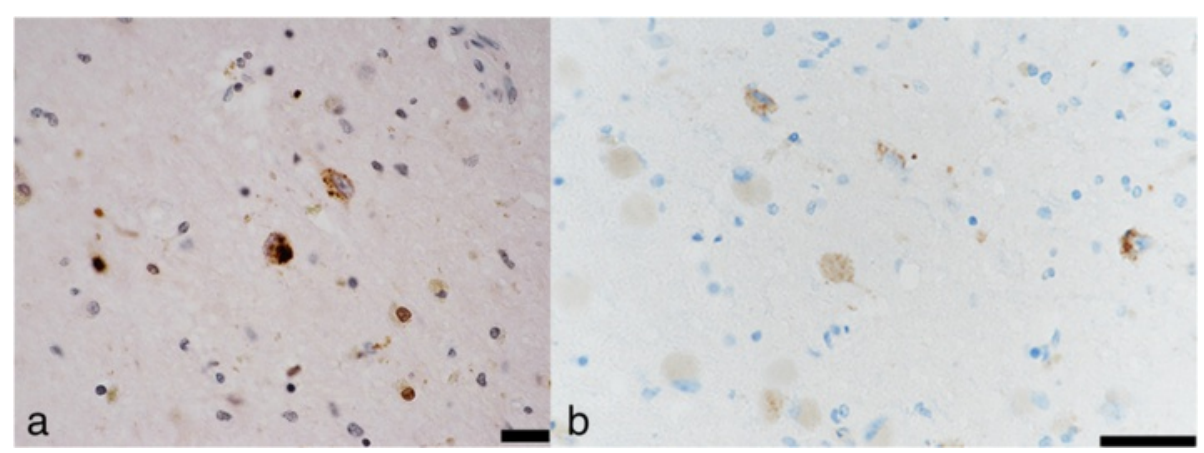

Fig. 6 TDP-43 pathology in the subiculum (a) and basal forebrain (b) from Case 1. Neuronal cytoplasmic inclusions and neurites are visible. TDP43 immunohistochemistry. Bar $=100 \mu \mathrm{m}$ (a). Bar $=50 \mu \mathrm{m}$ (b)

(Table 1, Figs. 3b and 4b). Alpha-synuclein immunoreactive deposits and hippocampal sclerosis were not observed, and there were no AT8-immunoreactive tufted astrocytes or astrocytic plaques. ARTAGs were observed in the following regions: 1) subpial/lobar, subcortical/frontal, and basal forebrain, 2) gray matter/subcortical/basal forebrain, and 3) white matter/lobar/lateral temporal (Figs. 7 and 8). The astrocytic tau deposits in the frontal cortex were not associated with $A ß$ deposits. TDP-43-immunoreactive NCIs and neuritis were moderately observed in the hippocampus and subiculum, and GCIs were also seen (Table 2). Mild to moderate arteriolosclerosis was observed (Table 3). The basal ganglia exhibited severe état criblé (Fig. 9b).

\section{Case 3}

\section{Gross neuropathology}

The fresh brain from Case 3 weighed 1015 grams. After formalin fixation, mild atrophy at the frontal and temporal lobes was observed (Fig. 1c). Atherosclerosis was mild in the major cerebral arteries, and no atheromatous plaques were seen in the leptomeningeal vessels. On coronal sections, there were no focal lesions, but mild enlargement of the lateral ventricle was observed (Fig. 2c). Subcortical nuclei were well preserved without obvious atrophy, and atrophy was not observed in the cerebellum. The substantia nigra and locus coeruleus were well pigmented.

\section{Microscopic neuropathology}

Neuronal loss and gliosis was none to mild in the majority of the cerebral cortex. Neurons in the substantia nigra and locus coeruleus were well preserved. The level of $A ß$-immunoreactive neuritic and diffuse plaques was determined to be sparse according to CERAD methodology. Aß-immunoreactive parenchymal deposits were deemed phase 1 using Thal's methodology. No Aß-immunoreactive cerebral amyloid angiopathy was observed, and AT8-immunoreactive NFTs were stage III according to Braak methodology. Therefore, Case 3 was assigned

Table 2 Severity and distribution of TDP-43-immunoreactive deposition

\begin{tabular}{|c|c|c|c|c|c|c|c|c|c|c|c|c|c|c|c|c|}
\hline \multirow[t]{2}{*}{ Areas } & \multicolumn{4}{|l|}{ Case 1} & \multicolumn{4}{|l|}{ Case 2} & \multicolumn{4}{|c|}{ Case 3} & \multicolumn{4}{|c|}{ Case 4} \\
\hline & $\mathrm{NCl}$ & Neurites & NNI & $\mathrm{GCl}$ & $\mathrm{NCl}$ & Neurites & $\mathrm{NNI}$ & $\mathrm{GCl}$ & $\mathrm{NCl}$ & Neurites & $\mathrm{NNI}$ & $\mathrm{GCl}$ & $\mathrm{NCl}$ & Neurites & NNI & $\mathrm{GCl}$ \\
\hline Basal ganglia & $\begin{array}{l}\text { Basal forebrain; } \\
+++\end{array}$ & $\begin{array}{l}\text { Basal } \\
\text { forebrain; + }\end{array}$ & - & + & - & - & - & - & - & - & - & - & - & - & - & - \\
\hline Temporal lobe & - & - & - & - & - & - & - & - & - & - & - & - & - & - & - & - \\
\hline Uncus & - & ++ & - & + & - & - & - & - & - & + & - & - & - & - & - & $+/-$ \\
\hline $\begin{array}{l}\text { Hippocampus- } \\
\text { PHG }\end{array}$ & $\begin{array}{l}\text { CA } 1 ;+++ \\
\text { Subiculum; +++ } \\
\text { Dentate fascia; } \\
+/-\end{array}$ & $\begin{array}{l}\text { CA1; +++ } \\
\text { Subiculum; } \\
+++\end{array}$ & + & + & $\begin{array}{l}\text { CA1;++ } \\
\text { subiculum++ } \\
\text { Dentate } \\
\text { fascia;+/- }\end{array}$ & $\begin{array}{l}\text { CA1; ++ } \\
\text { subiculum } \\
++\end{array}$ & - & + & - & - & - & - & - & - & - & - \\
\hline Motor cortex & - & - & - & - & - & - & - & - & - & - & - & - & - & - & - & - \\
\hline Midbrain & - & - & - & - & - & - & - & - & - & - & - & - & - & - & - & - \\
\hline Medulla & - & - & - & - & - & - & - & - & - & - & - & - & - & - & - & - \\
\hline Spinal cord & - & - & - & - & - & - & - & - & - & - & - & - & - & - & - & - \\
\hline
\end{tabular}

Abbreviations: $\mathrm{GCl}$ glial cytoplasmic inclusions, $\mathrm{NCl}$ neuronal cytoplasmic inclusion, $\mathrm{NNI}$ neuronal nuclear inclusion, $\mathrm{PHG}$ parahippocampal gyrus -: none

+ : mild

++ : moderate

+++ : severe 
Table 3 Small vessel disease and white matter rarefaction

\begin{tabular}{|c|c|c|c|c|c|c|c|c|}
\hline & \multicolumn{4}{|c|}{ Arteriolosclerosis } & \multicolumn{4}{|c|}{$\begin{array}{l}\text { White matter rarefaction } \\
\text { état criblé (basal ganglia and thalamus) }\end{array}$} \\
\hline & Case 1 & Case 2 & Case 3 & Case 4 & Case 1 & Case 2 & Case 3 & Case 4 \\
\hline Frontal & 2 & 2 & 1 & 2 & 2 & 0 & 1 & 2 \\
\hline Temporal & 1 & 2 & 1 & 2 & 2 & 0 & 0 & 2 \\
\hline Parietal & 2 & 1 & 2 & 1 & 2 & 1 & 1 & 1 \\
\hline Occipital & 2 & 1 & 0 & 2 & 2 & 0 & 0 & 1 \\
\hline Hippocampus & 1 & 1 & 1 & 1 & 1 & 0 & 1 & 1 \\
\hline Basal ganglia & 2 & 2 & 2 & 2 & 2 & 3 & 3 & 2 \\
\hline Thalamus & 2 & 2 & 2 & 2 & 2 & 2 & 2 & 2 \\
\hline Cerebellum & 2 & 2 & 2 & 2 & 0 & 1 & 0 & 1 \\
\hline
\end{tabular}

Instead of white matter rarefaction, the severity of état criblé was analyzed in the basal ganglia and thalamus

0: none, 1: mild, 2: moderate, 3: severe

an unclassified level of AD pathological changes according to NIA-Reagan criteria and low level according to NIA-AA criteria (Table 1, Figs. 3c and 4c). This pathological pattern was consistent with "PART possible" (NFT stage 3, Thal phase 1) [15]. ARTAGs were classified as follows: 1) subpial/MTL/uncus, 2) gray matter/ MTL and subcortical/hippocampus and basal forebrain, and 3) white matter/MTL/uncus (Figs. 7 and 10). There were also no AT8-immunoreactive tufted astrocytes or astrocytic plaques, as well as no alpha-synucleinimmunoreactive deposits, and no hippocampal sclerosis was observed. Some TDP-43-immunoreactive neuritis was sparsely seen in the uncus (Table 2). Mild to moderate arteriolosclerosis and état criblé were also observed (Fig. 9c, Table 3).

\section{Case 4 \\ Gross neuropathology}

The fresh brain of Case 4 weighed 1115 grams. After formalin fixation, mild atrophy in the temporal lobe was observed (Fig. 1d). Mild atherosclerosis was seen in the major cerebral arteries, but atheromatous plaques were not observed in the leptomeningeal vessels. On coronal sections, focal lesions were not observed, but there was mild enlargement of the lateral ventricle. Although the subcortical nuclei were

\begin{tabular}{|c|c|c|c|c|c|c|}
\hline \multirow[t]{2}{*}{ Step 1} & \multirow{2}{*}{\begin{tabular}{|l|} 
Step 2 \\
$\begin{array}{l}\text { Major anatomical } \\
\text { involvement }\end{array}$ \\
\end{tabular}} & \multicolumn{5}{|c|}{ Step 4} \\
\hline & & \multicolumn{4}{|c|}{ Detailed regional distribution and studied on pathogenesis } & $\begin{array}{l}1 \text { case } \\
2 \text { cases }\end{array}$ \\
\hline \multirow{4}{*}{ Subpial } & MTL & Inf. Temporal gy. & Hippocampus & Amygdala & Uncus & \\
\hline & Lobar & Frontal & Parietal & Occipital & Lat. Te & oral \\
\hline & Subcortical & Basal forebrain & & & & \\
\hline & Brainstem & Mesencephalopn & Pons & Medulla & & \\
\hline \multirow{4}{*}{ Subependymal } & MTL & Temporal horn & & & & \\
\hline & Lobar & LV. Frontal horn & LV,. Occipital horn & & & \\
\hline & Subcortical & LV. Caudate & 3V. Thalamus & & & \\
\hline & Brainstem & Aq. Mesencephalon & Aq. Pons & Aq. Medulla & & \\
\hline \multirow{4}{*}{ Gray matter } & MTL & Inf. Temporal gy. & Hippocampus & Amygdala & & \\
\hline & Lobar & Frontal & Parietal & Occipital & \multicolumn{2}{|c|}{ Lat. Temporal } \\
\hline & Subcortical & Accumbens & Caud/Put & GP & \multicolumn{2}{|c|}{ Basal forebrain } \\
\hline & Brainstem & Substantia nigra & Pons & Medulla & & \\
\hline \multirow{4}{*}{ White matter } & MTL & Inf. Temporal gy. & Hippocampus & Amygdala & \multicolumn{2}{|l|}{ uncus } \\
\hline & Lobar & Frontal & Parietal & Occipital & \multicolumn{2}{|c|}{ Lat. Temporal } \\
\hline & Subcortical & Int. capsule & Subinsular & Pencil fiber & & \\
\hline & Brainstem & Cerebral peduncli & Pyramids & Midline & & \\
\hline \multirow{4}{*}{ Perivascular } & MTL & Inf. Temporal gy. & Hippocampus & Amygdala & & \\
\hline & Lobar & Frontal & Parietal & Occipital & \multicolumn{2}{|c|}{ Lat. Temporal } \\
\hline & Subcortical & Accumbens & Caud/Put & GP & \multicolumn{2}{|c|}{ Basal forebrain } \\
\hline & Brainstem & Mesencephalopn & Pons & Medulla & & \\
\hline
\end{tabular}

Fig. 7 Distribution and severity of ARTAG pathology in four cases. The basal forebrain is a relatively commonly affected area 


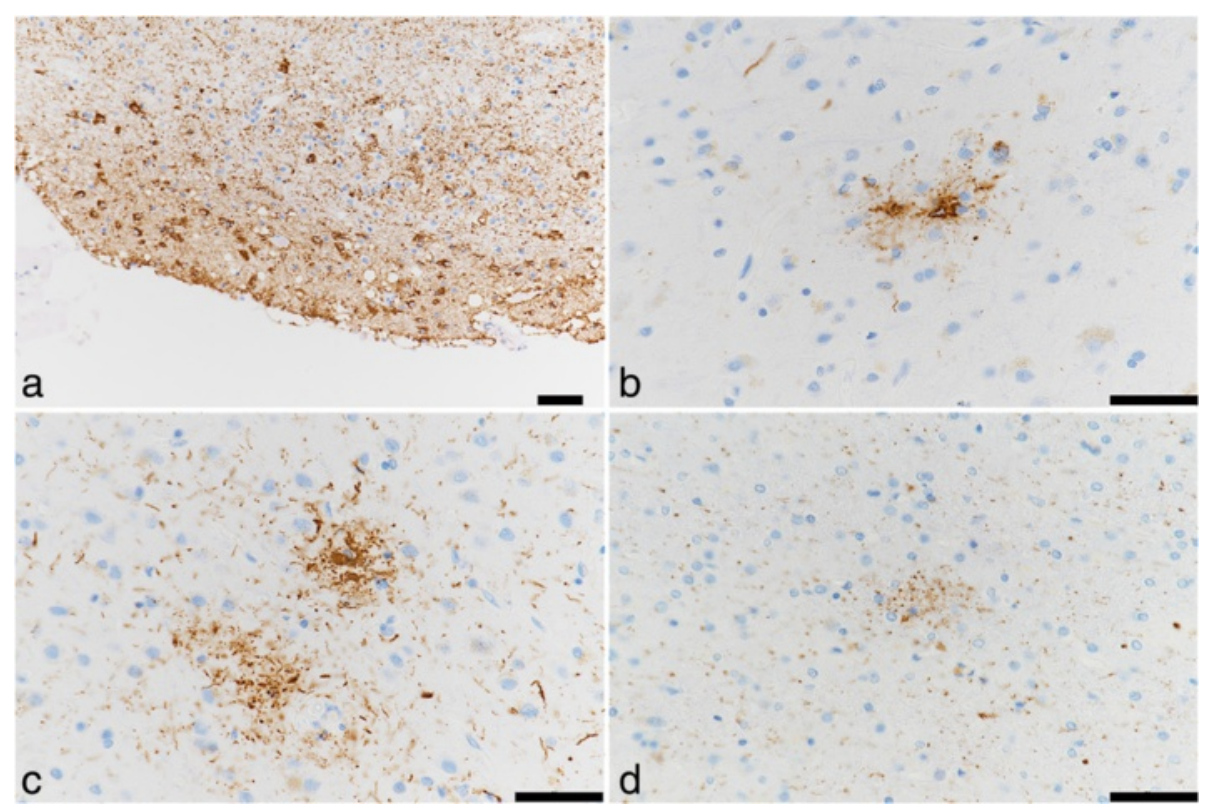

Fig. 8 ARTAG of Case 2. ARTAG (thorn-shaped astrocytes) is present in subpial areas of the basal forebrain (a). Granular or fuzzy astrocytes (GFA) are seen in the frontal (b) and occipital (c) cortex. In rare instances, possible GFAs are seen in the temporal white matter (d). Immunohistochemistry using monoclonal antibody specific to p-tau (AT8). Bar $=50 \mu \mathrm{m}$

well preserved, there were some small lacunes in the basal ganglia. No atrophy was seen in the cerebellum, and the substantia nigra and locus coeruleus were well pigmented. A small parenchymal hemorrhage $(<3 \mathrm{~mm})$ was present in the middle pontine base.

\section{Microscopic neuropathology}

Neuronal loss and gliosis was none to mild in the majority of the cerebral cortex. Neurons in the substantia nigra and locus coeruleus were well preserved. Aß-immunoreactive neuritic and diffuse plaque scores were moderate and frequent according to CERAD methodology, respectively. Aß-

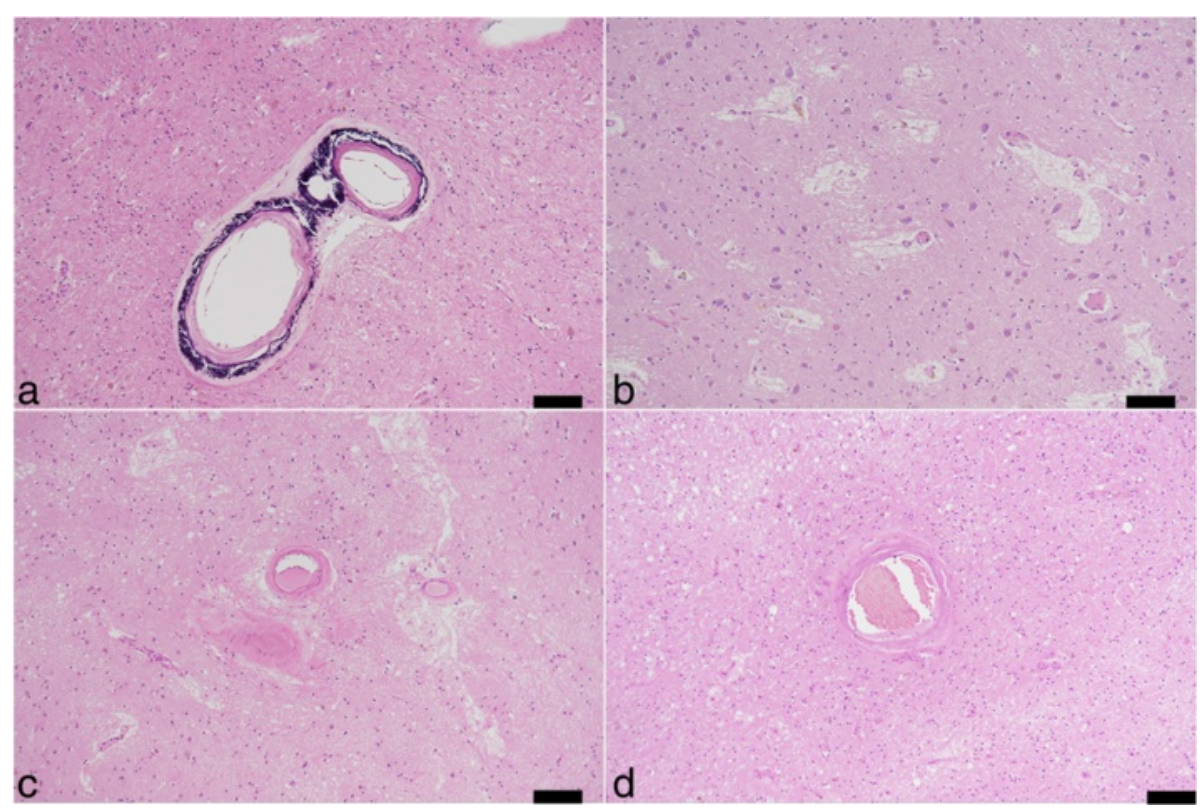

Fig. 9 Representative photomicrographs of small-vessel disease (arteriolosclerosis) and état criblé (a-d). Mineralization is seen in Case 1 (a). État crible is seen in the basal ganglia and thalamus (b and c). a Case 1: globus pallidus, (b) Case 2: thalamus, (c) Case 3: putamen, (d) Case 4: globus pallidus. Bar $=50 \mu \mathrm{m}$ 


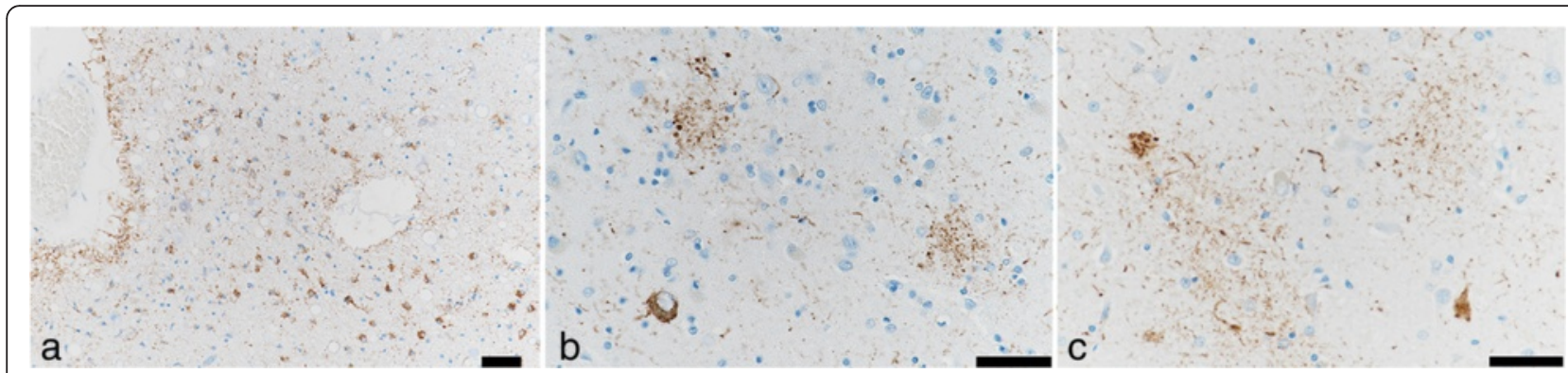

Fig. 10 ARTAG of Case 3. ARTAG (thorn-shaped astrocytes) is present in white matter close to the hippocampus (a). GFAs are seen in gray matter of the basal forebrain (b) and CA4 (c). Immunohistochemistry using monoclonal antibody specific to p-tau (AT8). Bar $=50 \mu \mathrm{m}$

immunoreactive parenchymal deposits were classified as phase 2 according to Thal's methodology. Aß-immunoreactive cerebral amyloid angiopathy was mildly observed in the parenchymal and leptomeningeal small vessels in the occipital lobe, and AT8-immunoreactive NFTs were considered stage IV using Braak methodology. Therefore, Case 4 was assigned an intermediate level of $\mathrm{AD}$ pathological changes according to NIA-Reagan and NIA-AA criteria. There were no AT8-immunoreactive tufted astrocytes or astrocytic plaques. ARTAGs were classified as follows: 1) subpial/subcortical/basal forebrain, 2) subependymal/MTL/ temporal lobes, subependymal/lobar/LV of occipital horn, 3) gray matter/lobar/frontal, and 4) perivascular/subcortical/basal forebrain. In all four cases, ARTAG was strongly immunoreactive with AT8 (Figs. 7 and 11). In some instances, ARTAG was also immunoreactive to RD4 antibody, but less depicted by the Gallyas-Braak staining (Fig. 11). This ARTAG immunoreactivity was similar to
Cases 1, 2 and 3. Alpha-synuclein-immunoreactive deposits and hippocampal sclerosis were not observed. TDP-43immunoreactive GCIs were sparsely observed in the uncus (Table 2), and mild to moderate arteriolosclerosis was observed (Fig. 9d, Table 3).

\section{Discussion}

The present study provides neuropathological results from four supercentenarians ( $\geq 110$ years of age) using conventional and immunohistochemical methods. We emphasize that this novel study is the first unique opportunity to comprehensively determine neuropathological conditions in four supercentenarians. We also introduce NIA diagnostic methodology for Alzheimer's disease, revealing TDP-43 and ARTAG pathology in those cases. Compared with centenarians, there are currently approximately 50 living supercentenarians in the world (www.grg.org, last updated, July 14, 2016). We

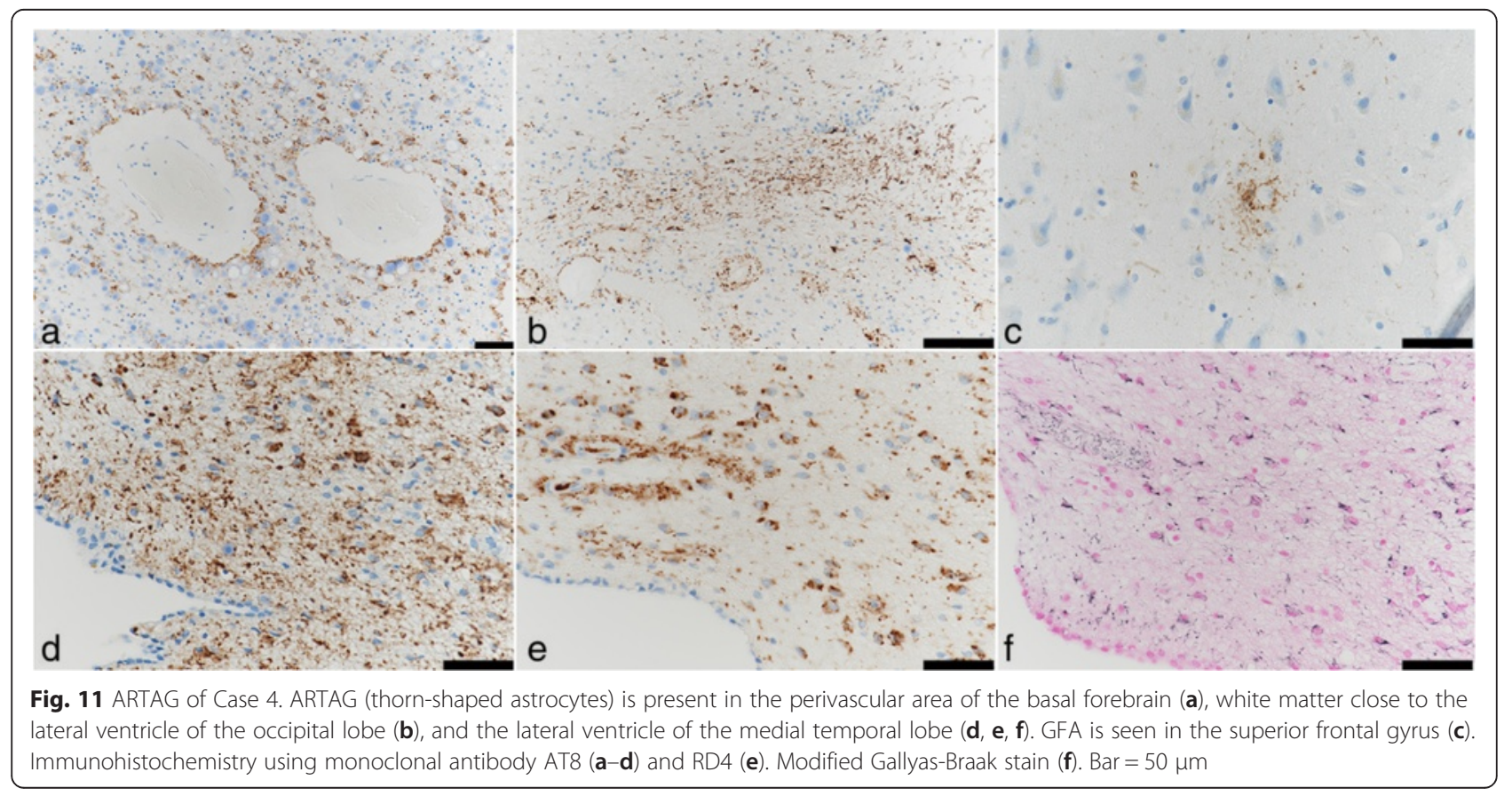


believe that supercentenarians are exceptionally distinct human beings, and the study of this cohort is important for understanding the mechanisms of successful aging.

\section{Clinical information}

Because our studies did not perform precise neurological or neuropsychological studies, it was difficult to clinically determine whether the four cases experienced dementia. However, the cases exhibited a certain degree of independence during the last stages of life.

Gender and race might play a role in human longevity. As shown in our results, all four cases were Japanese woman. According to data from the Gerontology Research Group (www.grg.org, last updated, July 14, 2016), most living supercentenarians are women (45/47 individuals). Even in the deceased supercentenarian cohort, the number of men is low, suggesting that it is more difficult for men to reach the supercentenarian age.

Women are considered to be at higher risk of Alzheimer's disease than men $[16,17]$. In previous studies, the rate of dementia increases in women aged $>90$ years [18-21]. In a 90+ study, the incidence of dementia increased exponentially with age, reaching $40.7 \%$ (10.9 \% men, $38.3 \%$ women) in centenarians [17]. However, gender differences were not observed $\geq 90$ years of age [22]. In one study, the incidence of dementia decreased after 97 years of age in women [23].

In centenarian studies, the prevalence of dementia was 27-79 \% [24-31]. Conversely, other studies have shown that $37 \%$ of centenarians do not exhibit dementia [32] and may have high cognitive function [24,33]. This discrepancy between dementia rates may be associated with different methods and samples, as well as selection biases [24]. However, researchers should take care with the relatively low prevalence of dementia in those centenarians as there may be some underestimation [18].

Although four supercentenarians were not clinically considered to exhibit dementia conditions in the present study, they had low MMSE scores. However, visual and hearing impairments pose problems when applying the MMSE test for extremely old individuals [32]. In fact, Case 1 refused to undergo the MMSE test at the time of interview. This suggests that a discrepancy might be present between MMSE and CDR scores in centenarians [32]. Whether MMSE was appropriate for this individual remains unclear. This was further complicated by the fact that Cases 2 and 3 were classified as dementia based on only MMSE scores. Although neuropathological alterations in these four cases likely played a role in cognitive impairment and motor dysfunction, a more precise prospective analysis is needed for extremely old individuals.

Although a previous case report of a female supercentenarian mentioned well-preserved cognitive function
[5], the definition of dementia in such extremely aged individuals remains unclear. At this point, we have not validated neuropsychological examinations for the extremely elderly or standard scores for commonly used tests, such as the Mini-Mental State Examination and Frontal Assessment Battery.

Although the Japanese population is considered to have long longevity, there is no strong scientific evidence that longevity is associated with a specific ethnicity or race. In Alzheimer's disease, many kinds of protective factors have been reported [34]. Although we questioned the relatives about lifestyle and diet factors, we were not able to identify specific factors. The individuals were very physically active and participated in social activities in a positive manner. Future prospective studies of supercentenarians are needed to recruit individuals in their 90 s and centenarians.

\section{Apo E genotype}

GWAS studies have shown that the ApoE gene is strongly associated with human longevity $[35,36]$. In particular, the $\varepsilon 2$ allele is enriched in centenarians, compared with $\varepsilon 4$ [37]. In the present study, the $\varepsilon 4$ allele was not detected in any of the cases. Although more cases are needed to substantiate our hypothesis, results from the present study suggests that it might be difficult for individuals expressing the $\varepsilon 4$ allele to reach a supercentenarian age. Because the $\varepsilon 2$ allele is considered to be important for protection of $A \beta$ deposition [38], results from two of our cases suggests that the $\varepsilon 2$ allele may affect brain pathology.

\section{Neuropathology}

The neuropathological results from the present study provide unique and imperative findings of brain conditions in supercentenarians. The well-preserved brain shapes and weights in the gross neuropathological findings were surprising. In all cases, there was only mild atrophy in the frontal and/or temporal lobes. Previous results have shown that brain weight is affected by the presence of brain diseases, as well as individual height and gender [39]. Although we could not evaluate the exact height of each individual, the cases were relatively small or of average stature for aged Japanese females. Based on our previous analysis, the mean brain weight of female centenarians (mean age of 101) was 1066 grams (American Association of Neuropathologists, Inc., The 91st Annual Meeting, 2015 Denver, CO. Abstract No. 129). A recent U.S. study also reported that the unfixed brain weight of centenarians is 1000 to 1200 grams [40]. Unfortunately, there is no previous information for the average brain weight of supercentenarians. Nevertheless, we believe that brain weight in these four 
supercentenarians, in particular Cases 3 and 4, was relatively well-preserved.

Mild atherosclerosis of the major cerebral arteries might be a characteristic feature of supercentenarians. In Case 1, multiple small cortical infarcts with occluded leptomeningeal vessels were observed. Although those infarcts were possibly due to embolic stroke, we were not able to identify the embolism source. Once an individual experiences a major cerebrovascular accident or atherosclerosis of the intracranial cerebral arteries, the clinical prognosis is typically not good [41]. In fact, stroke is one of the most important causes of death and disability worldwide [42]. Results from this study suggest that well-preserved intracranial arteries might be an important element associated with human longevity.

The microscopic findings were also impressive in the four cases. None of them exhibited high-likelihood levels of AD pathology. In one case (Case 3), only low levels of AD changes were observed (Table 1). As stated earlier, age and female sex are generally considered to be risk factors for developing $\mathrm{AD}[16,17,40,43]$. Further, females typically live longer than males, which could increase the risk for developing AD. However, this universal concept has not been extrapolated to supercentenarians, who might have a biological condition that protects against increased tau and $A \beta$ accumulation in the brain.

Primary age-related tauopathy (PART) was recently introduced to describe a pathology commonly observed in the brains of aged individuals [3]. PART is microscopically defined as NFTs with Braak stage $\leq$ IV (usually III or lower) and Thal $A \beta$ phase 0 (definite) or $1-2$ (possible) $[3,15,44]$. In the present study, one case (Case 3) was assigned PART possible. Studies have discussed whether PART is an independent condition or a characteristic of AD [44-46]. At least in our study of PART supercentenarians, there were very few neuritic plaques, and the plaques exhibited an early Thal's phase of $A ß$ deposition, even at the age of 114 years. It is not currently possible to determine whether extremely aged individuals will also suffer from AD in the future. Further analysis of these brains could provide a better understanding as to how humans can protect against the progression of $A D$ pathology.

ARTAG was recently determined to be a pathological astrocytic condition involving tau accumulation [14]. ARTAGs include not only thorn-shaped astrocytes and granular or fuzzy astrocytes, but also other forms of ARTAG that do not include disease-specific astrocytic tau pathologies, such as tufted astrocytes, astrocytic plaques, ramified astrocytes, or globular astrocytic tau. In our supercentenarians, the medial temporal lobes and basal forebrain were relatively common areas for ARTAGs (Fig. 6), and in those areas, most ARTAGs were thorn-shaped astrocytes. However, ARTAGs were also observed in other brain regions. Nevertheless, the role of ARTAGs remains poorly understood. Because common neurodegenerative pathology is limited in supercentenarians, the presence of ARTAGs could affect clinical and neurological conditions of supercentenarians. However, further analysis is necessary to clarify ARTAG pathology in supercentenarians.

TDP-43 deposition is emphasized in the hippocampus and parahippocampus in aged individuals [2]. Basal forebrain TDP-43 pathology is also strongly associated with hippocampal sclerosis-related aging, which is one of the most important pathological changes indicating dementia $[2,3,47,48]$ and AD pathology $[49,50]$ in aged individuals. TDP-43 pathology of the hippocampus and parahippocampus was observed in two out of four cases. In Case 1, TDP-43 pathology was only observed in the basal forebrain. Again, hippocampal sclerosis or AD was not diagnosed in the four supercentenarians. Although TDP-43 pathology has been reported in the uncus in normal aging individuals [51], our cases exhibited no severe TDP-43 pathology in the uncus. Additionally, although TDP-43 pathology is considered to spread in a hierarchical manner in individuals with motor neuron disease or frontotemporal lobar degeneration [52, 53], our pathological results did not suggest either of these diseases. Therefore, TDP-43 deposition in our cases could be associated with the aging processes, although the changes were relatively mild. Further studies are needed to determine the pathological changes of TDP43 in supercentenarian brains.

Arteriolosclerosis was consistently observed, but it was not very severe in the four cases (Table 3). A recent study mentioned that brain arteriolosclerosis could be associated with TDP-43 deposition in the amygdala and limbic system, as well as hippocampal sclerosis [3]. This pathological condition was termed "cerebral age-related TDP-43 pathology and arteriolosclerosis" (CARTS) [3]. Based on this concept, Cases 1 and 2 were at the early clinical stage of CARTS. However, Cases 3 and 4 were at the preclinical stage, and no hippocampal sclerosis was observed in all cases. Therefore, CARTS or hippocampus sclerosis-related aging pathology was a mild condition in the supercentenarians. Because the right cerebral hemispheres in all cases were stored as frozen tissue and were not analyzed, it is important to keep in mind that hippocampus sclerosis-related aging could exist in the right hemisphere.

There are some limitations in the present study. Because all individuals were only visited once or twice by one of the authors, we were not able to obtain detailed clinical information. Also, because neuroimaging studies were not performed, we could not report clinical, neuroradiological, or neuropathological correlations in the 
present study. We would like to emphasize, however, that it is difficult to obtain autopsies from such extremely old individuals, because they are usually placed in nursing homes or elderly care facilities in Japan.

\section{Conclusions}

Neuropathological alterations associated with aging were relatively mild to moderate in the supercentenarian brain. Considering their extremely old age, the individuals might have some neuroprotective factors against aging. In fact, $\mathrm{AD}$ pathological changes of NIA-Reagan and NIA-AA criteria remained low to intermediate in all cases. Future prospective studies and extensive molecular analyses are needed to determine the mechanisms of human longevity. Because we have sufficient frozen tissue from these supercentenarians, we welcome supercentenarian research collaborations.

\section{Acknowledgements}

We are deeply grateful to all the study individuals and their relatives. We thank Mitsutoshi Tano, Katsura Suwabe, and Shoken Aizawa for technical support.

\section{Funding}

This study was supported in part by Grants-in-Aid for Scientific Research on Innovative Areas (Comprehensive Brain Science Network, 221S0003) (MT) and Platform of Supporting Cohort Study and Biospecimen Analysis (JSPS KAKENHI JP 16H06277) (MT), the Ministry of Education, Culture, Sports, Science and Technology, Japan. MT, HN, YA and MM were also supported in part by Keio University Program for Initiative Research Project, Longevity Initiative.

\section{Availability of data and materials}

All data generated or analyzed during this study are included in this published article.

\section{Authors' contributions}

MT: conceptualization, methodology, autopsy, investigation (neuropathological analysis), and writing manuscript; $\mathrm{NH}$ and $\mathrm{YA}$ : conceptualization of clinical study; BM: autopsy; MM: supervision, clinical study, and writing manuscript. All authors read and approved the final manuscript.

\section{Competing interests}

The authors declare that they have no competing interests.

\section{Consent for publication}

We obtained written informed consent from the deceased relatives for publication.

\section{Ethics approval and consent to participate}

All procedures performed in studies involving human participants were in accordance with the ethical standards of the institutional and/or national research committee and with the 1964 Helsinki declaration and its later amendments or comparable ethical standards. We obtained written informed consent from the deceased relatives for autopsy and further neuropathological analysis, and all subjects were registered with our brain bank for future research. The brain bank was approved by the Ethics Committee of Mihara Memorial Hospital for neuropathological analysis $(072-01,078-01)$

\section{Author details}

'Department of Neurology, Saitama Medical University International Medical Center, Yamane, 1397-1 Yamane, Hidaka, Saitama 350-1298, Japan. ${ }^{2}$ Center for Supercentenarian Medical Research, School of Medicine, Keio University, 35 Shinanomachi, Shinjuku-ku, Tokyo 160-8582, Japan. ${ }^{3}$ Department of
Neurology and Brain Bank, Mihara Memorial Hospital, 366 Ohtemachi, Isesaki, Gunma 372-0006, Japan. " Department of Neuropsychiatry, School of Medicine, Keio University, 35 Shinanomachi, Shinjuku-ku, Tokyo 160-8582, Japan.

Received: 17 August 2016 Accepted: 17 August 2016

Published online: 02 September 2016

\section{References}

1. Mizutani T, Shimada H. Neuropathological background of twenty-seven centenarian brains. J Neurol Sci. 1992;108:168-77.

2. Neltner JH, Abner EL, Jicha GA, et al. Brain pathologies in extreme old age. Neurobiol Aging. 2016;37:1-11.

3. Nelson PT, Trojanowski JQ, Abner EL, et al. "New Old Pathologies": AD, PART, and Cerebral Age-Related TDP-43 With Sclerosis (CARTS). J Neuropathol Exp Neurol. 2016;75:482-98.

4. Bateman RJ, Xiong C, Benzinger TL, et al. Clinical and biomarker changes in dominantly inherited Alzheimer's disease. N Engl J Med. 2012;367:795-804.

5. den Dunnen WF, Brouwer WH, Bijlard E, et al. No disease in the brain of a 115-year-old woman. Neurobiol Aging. 2008;29:1127-32.

6. Takao M, Ghetti B, Yoshida H, et al. Early-onset dementia with Lewy bodies. Brain Pathol. 2004;14:137-47.

7. Takao M, Ghetti B, Hayakawa I, et al. A novel mutation (G217D) in the Presenilin 1 gene (PSEN1) in a Japanese family: presenile dementia and parkinsonism are associated with cotton wool plaques in the cortex and striatum. Acta Neuropathol. 2002;104:155-70.

8. Mirra SS, Heyman A, McKeel D, et al. The Consortium to Establish a Registry for Alzheimer's Disease (CERAD). Part II. Standardization of the neuropathologic assessment of Alzheimer's disease. Neurology. 1991;41: 479-86.

9. Braak H, Alafuzoff I, Arzberger T, Kretzschmar H, Del Tredici K. Staging of Alzheimer disease-associated neurofibrillary pathology using paraffin sections and immunocytochemistry. Acta Neuropathol. 2006;112:389-404.

10. Braak H, Braak E. Neuropathological stageing of Alzheimer-related changes. Acta Neuropathol. 1991;82:239-59.

11. Thal DR, Rub U, Orantes M, Braak H. Phases of A beta-deposition in the human brain and its relevance for the development of AD. Neurology. 2002; 58:1791-800.

12. Hyman BT, Phelps $\mathrm{CH}$, Beach $\mathrm{TG}$, et al. National Institute on AgingAlzheimer's Association guidelines for the neuropathologic assessment of Alzheimer's disease. Alzheimers Dement. 2012;8:1-13.

13. Montine TJ, Phelps $\mathrm{CH}$, Beach TG, et al. National Institute on AgingAlzheimer's Association guidelines for the neuropathologic assessment of Alzheimer's disease: a practical approach. Acta Neuropathol. 2012;123:1-11.

14. Kovacs GG, Ferrer I, Grinberg LT, et al. Aging-related tau astrogliopathy (ARTAG): harmonized evaluation strategy. Acta Neuropathol. 2016;131:87-102.

15. Crary JF, Trojanowski JQ, Schneider JA, et al. Primary age-related tauopathy (PART): a common pathology associated with human aging. Acta Neuropathol. 2014;128:755-66.

16. Vina J, Lloret A. Why women have more Alzheimer's disease than men: gender and mitochondrial toxicity of amyloid-beta peptide. J Alzheimers Dis. 2010;20 Suppl 2:S527-33.

17. Corrada MM, Brookmeyer R, Paganini-Hill A, Berlau D, Kawas CH. Dementia incidence continues to increase with age in the oldest old: the 90+ study. Ann Neurol. 2010;67:114-21.

18. The Canadian Study of Health and Aging Working Group. The incidence of dementia in Canada. Neurology. 2000;55:66-73.

19. Edland SD, Rocca WA, Petersen RC, Cha RH, Kokmen E. Dementia and Alzheimer disease incidence rates do not vary by sex in Rochester. Minn Arch Neurol. 2002:59:1589-93.

20. Hall CB, Verghese J, Sliwinski M, et al. Dementia incidence may increase more slowly after age 90: results from the Bronx Aging Study. Neurology. 2005:65:882-6.

21. Ruitenberg A, Ott A, van Swieten JC, Hofman A, Breteler MM. Incidence of dementia: does gender make a difference? Neurobiol Aging. 2001;22:575-80

22. Yang Z, Slavin MJ, Sachdev PS. Dementia in the oldest old. Nat Rev Neurol. 2013:9:382-93.

23. Miech RA, Breitner JC, Zandi PP, Khachaturian AS, Anthony JC, Mayer L. Incidence of AD may decline in the early 90s for men, later for women: The Cache County study. Neurology. 2002;58:209-18. 
24. Kliegel M, Moor C, Rott C. Cognitive status and development in the oldest old: A longitudinal analysis from the Heidelberg Centenarian Study. Arch Gerontol Geriatr. 2004;39:143-56.

25. Samuelsson SM, Alfredson BB, Hagberg B, Samuelsson G, Nordbeck B, Brun A. The Swedish Centenarian Study: a multidisciplinary study of five consecutive cohorts at the age of 100. Int J Aging Hum Dev. 1997;45:223-53.

26. Silver MH, Jilinskaia E, Perls TT. Cognitive functional status of age-confirmed centenarians in a population-based study. J Gerontol B Psychol Sci Soc Sci. 2001;56:134-40.

27. Copeland JR, McCracken CF, Dewey ME, et al. Undifferentiated dementia, Alzheimer's disease and vascular dementia: age- and gender-related incidence in Liverpool. The MRC-ALPHA Study. Br J Psychiatry J Ment Sci. 1999:175:433-8.

28. Hagberg B, Bauer Alfredson B, Poon LW, Homma A. Cognitive functioning in centenarians: a coordinated analysis of results from three countries. J Gerontol Ser B Psychol Sci Soc Sci. 2001;56:P141-51.

29. Ravaglia G, Forti $P$, De Ronchi D, et al. Prevalence and severity of dementia among northern Italian centenarians. Neurology. 1999:53:416-8.

30. Sobel E, Louhija J, Sulkava R, et al. Lack of association of apolipoprotein E allele epsilon 4 with late-onset Alzheimer's disease among Finnish centenarians. Neurology. 1995:45:903-7.

31. Perls T. Dementia-free centenarians. Exp Gerontol. 2004;39:1587-93.

32. Andersen-Ranberg $K$, Vasegaard $L$, Jeune $B$. Dementia is not inevitable: a population-based study of Danish centenarians. J Gerontol Ser B Psychol Sci Soc Sci. 2001;56:P152-9.

33. Jopp DS, Park M-KS, Lehrfeld J, Paggi ME. Physical, cognitive, social and mental health in near-centenarians and centenarians living in New York City: findings from the Fordham Centenarian Study. BMC Geriatr. 2016;16:1-10.

34. Winblad B, Amouyel P, Andrieu S, et al. Defeating Alzheimer's disease and other dementias: a priority for European science and society. Lancet Neurol. 2016;15:455-532

35. Beekman $\mathrm{M}$, Blanche $\mathrm{H}$, Perola $\mathrm{M}$, et al. Genome-wide linkage analysis for human longevity: Genetics of Healthy Aging Study. Aging Cell. 2013;12:184-93.

36. Deelen J, Uh HW, Monajemi R, et al. Gene set analysis of GWAS data for human longevity highlights the relevance of the insulin/IGF-1 signaling and telomere maintenance pathways. Age (Dordr). 2013;35:235-49.

37. Ryu S, Atzmon G, Barzilai N, Raghavachari N, Suh Y. Genetic landscape of APOE in human longevity revealed by high-throughput sequencing. Mech Ageing Dev. 2016;155:7-9.

38. Ohm TG, Scharnagl H, Marz W, Bohl J. Apolipoprotein E isoforms and the development of low and high Braak stages of Alzheimer's disease-related lesions. Acta Neuropathol. 1999;98:273-80.

39. Skullerud K. Variations in the size of the human brain. Influence of age, sex, body length, body mass index, alcoholism, Alzheimer changes, and cerebral atherosclerosis. Acta Neurol Scand Suppl. 1985;102:1-94.

40. Filon JR, Intorcia AJ, Sue LI, et al. Gender Differences in Alzheimer Disease: Brain Atrophy, Histopathology Burden, and Cognition. J Neuropathol Exp Neurol. 2016;75:748-754.

41. Pu Y, Dou X, Liu L. Natural history of intracranial atherosclerotic disease. Front Neurol. 2014;5:125.

42. Bustamante A, García-Berrocoso T, Rodriguez N, et al. Ischemic stroke outcome: A review of the influence of post-stroke complications within the different scenarios of stroke care. Eur J Intern Med. 2016;29:9-21.

43. Riedel BC, Thompson PM, Brinton RD. Age, APOE and sex: Triad of risk of Alzheimer's disease. J Steroid Biochem Mol Biol. 2016;160:134-47.

44. Jellinger KA, Alafuzoff I, Attems J, et al. PART, a distinct tauopathy, different from classical sporadic Alzheimer disease. Acta Neuropathol. 2015:129:757-62

45. Duyckaerts C, Braak H, Brion JP, et al. PART is part of Alzheimer disease. Acta Neuropathol. 2015;129:749-56.

46. Weller RO, Hawkes CA, Carare RO, Hardy J. Does the difference between PART and Alzheimer's disease lie in the age-related changes in cerebral arteries that trigger the accumulation of Abeta and propagation of tau? Acta Neuropathol. 2015;129:763-6.

47. Nelson PT, Schmitt FA, Lin Y, et al. Hippocampal sclerosis in advanced age: clinical and pathological features. Brain. 2011;134:1506-18.

48. Cykowski MD, Takei H, Van Eldik L, et al. Hippocampal Sclerosis but Not Normal Aging or Alzheimer Disease Is Associated With TDP-43 Pathology in the Basal Forebrain of Aged Persons. J Neuropathol Exp Neurol. 2016;75:397-407.

49. Josephs KA, Murray ME, Whitwell JL, et al. Updated TDP-43 in Alzheimer's disease staging scheme. Acta Neuropathol. 2016;131:571-85.
50. Josephs KA, Whitwell JL, Weigand SD, et al. TDP-43 is a key player in the clinical features associated with Alzheimer's disease. Acta Neuropathol. 2014;127:811-24.

51. Uchino A, Takao M, Hatsuta $H$, et al. Incidence and extent of TDP-43 accumulation in aging human brain. Acta Neuropathol Commun. 2015;3:35.

52. Brettschneider J, Del Tredici K, Irwin DJ, et al. Sequential distribution of pTDP-43 pathology in behavioral variant frontotemporal dementia (bvFTD). Acta Neuropathol. 2014;127:423-39.

53. Brettschneider J, Del Tredici K, Toledo JB, et al. Stages of pTDP-43 pathology in amyotrophic lateral sclerosis. Ann Neurol. 2013;74:20-38.

\section{Submit your next manuscript to BioMed Central and we will help you at every step:}

- We accept pre-submission inquiries

- Our selector tool helps you to find the most relevant journal

- We provide round the clock customer support

- Convenient online submission

- Thorough peer review

- Inclusion in PubMed and all major indexing services

- Maximum visibility for your research

Submit your manuscript at www.biomedcentral.com/submit
Biomed Central 